\title{
On the Asymptotic Power of the Variance Ratio Test
}

\author{
Rohit S. Deo and Matthew Richardson \\ New York University
}

Abstract: The variance ratio test statistic, which is based on $k$-period differences of the data, is commonly used in empirical finance and economics to test the random walk hypothesis. We obtain the asymptotic power function of the variance ratio test statistic when the differencing period $k$ is increasing with the sample size $n$ such that $k / n \rightarrow \delta>0$. We show that the test is inconsistent against a variety of mean reverting alternatives, confirm the result in simulations, and then characterise the functional form of the asymptotic power in terms of $\delta$ and these alternatives.

\section{Introduction}

The random walk is one of the most important models in empirical finance and economics and several tests have been developed to detect deviations from this model. Parsimonious tests of this model have been developed for low frequency autocorrelations in the data, such as slow mean reverting processes, by employing multiple horizon data. One of the popular tests of this genre is based on the variance ratio $(V R)$ statistic, which is the sample variance of $k$-period differences, $x_{t}-x_{t-k}$, of the time series $x_{t}$, divided by $k$ times the sample variance of the first difference, $x_{t}-x_{t-1}$, for some integer $k$. Under the null hypothesis that the series $x_{t}$ is a random walk, the corresponding population 
variance ratio, given by $\operatorname{PVR}(k)=\operatorname{Var}\left(x_{t}-x_{t-k}\right) /\left[k \operatorname{Var}\left(x_{t}-x_{t-1}\right)\right]$, is 1 for any value of $k$. Arguably, the $V R$ statistic has become the primary tool for testing the random walk hypothesis for low frequency series. (See, for example, Campbell and Mankiw (1987), Cochrane (1988), Lo and Mackinlay (1988) and Poterba and Summers (1988) among many others). The use of the $V R$ statistic can be advantageous when testing against several interesting alternatives to the random walk model, most notably those hypotheses associated with mean reversion. In fact, a number of authors (e.g., Lo and Mackinlay (1989), Faust (1992) and Richardson and Smith (1991)) have found that the $V R$ statistic has optimal power against such alternatives. ${ }^{1}$

The practical implementation of the $V R$ statistic is, however, less straightforward. The statistical significance has been assessed using conventional asymptotic theory, that is, for fixed $k$ and the sample size $n$ increasing to infinity. Unfortunately, the underlying asymptotic theory provides a poor approximation to the small sample distribution of the $V R$ statistic. In fact, rather than being normally distributed (when standardised by $\sqrt{n}$ ), the statistics are severely biased and right skewed for large $k$, which makes application of the statistic problematic. A seemingly attractive solution to this problem is to use the result of Richardson and Stock (1989). They derived the asymptotic distribution of the $V R$ statistic under the random walk null, assuming that both $k$ and $n$ increase to infinity but in such a way that $k / n$ converges to a positive constant $\delta$ which is strictly less than 1 . They showed that the $V R$ statistic, without any normalization, converges to a functional of Brownian motion. This new distribution provides a far more robust approximation to the small sample distribution of the $V R$ statistic. Most current applications of the $V R$ statistic cite the $k / n \rightarrow \delta$ result as justification for using Monte Carlo distributions (i.e., set at $k=\delta n$ ) as representative of the $V R$ statistic's sampling distribution.

\footnotetext{
${ }^{1}$ Richardson and Smith (1991) and Daniel (2001) explore a wider range of possible test statistics.
} 
This paper provides another look at the $V R$ statistic and its statistical properties. ${ }^{2}$ In particular, we argue that the $k / n \rightarrow \delta$ result has some very poor properties under a wide class of interesting alternatives. Similar to the distribution under the null hypothesis of a random walk, for mean reverting alternatives with some random walk component, the $k / n \rightarrow \delta$ asymptotic theory also implies nondegenerate limiting distributions. Thus, even with a large sample size, the $V R$ statistic should not be expected to provide decisive evidence. Loosely speaking, in terms of the asymptotic theory, there is a trade-off between size and power properties when using the fixed $k$ asymptotics versus the $k / n \rightarrow \delta$ asymptotics.

The paper is organized as follows. In section 2, we define the $V R$ statistic, review the exiting literature on its asymptotic distribution, and provide the main theoretical result regarding its lack of power. Section 3 then explores some of the practical implications of this result, in particular, through simulation evidence for relevant alternative models to the random walk theory.

\section{The Variance Ratio Statistic's Power}

Given $n+1$ observations $x_{0}, x_{1}, \ldots, x_{n}$ of a time series, the variance ratio statistic with a positive integer $k(<n)$ as differencing period is defined as

$$
V R(k)=\hat{\sigma}_{b}^{2}(k) / \hat{\sigma}_{a}^{2}(k)
$$

where

$$
\hat{\sigma}_{b}^{2}(k)=\frac{n}{k(n-k+1)(n-k)}_{t=k}^{X^{n}}\left(x_{t}-x_{t-k}-k \hat{\mu}\right)^{2},
$$

\footnotetext{
2 Perron and Vodounou (2001) also looks at the VR statitistic's properties under the Richardson and Stock (1989) framework. Their approach, however, is different to the extent they keep the data span, $\mathrm{n}$, fixed and then take a continuoustime limit within this span. In other words, rather than assuming $n \rightarrow \infty$, they assume the sampling frequency of the data goes to zero.
} 


$$
\hat{\sigma}_{a}^{2}(k)=\frac{1}{n-1}_{t=1}^{X^{n}}\left(x_{t}-x_{t-1}-\hat{\mu}\right)^{2}
$$

and

$$
\hat{\mu}=n_{t=1}^{-1}\left(x_{t}-x_{t-1}\right) .
$$

In the usual fixed $k$ asymptotic treatment, under the null hypothesis that the $\left\{x_{t}\right\}$ follow a random walk with possible drift, given by

$$
x_{t}=\mu+x_{t-1}+\varepsilon_{t}
$$

where $\mu$ is a real number and $\left\{\varepsilon_{t}\right\}$ is a sequence of zero mean independent random variables, it is possible to show (see, for example, Lo and Mackinlay (1988)) that

$$
\sqrt{n}(V R(k)-1) \stackrel{D}{\rightarrow} N^{3} 0, \sigma_{k}^{2}
$$

where $\sigma_{k}^{2}$ is some simple function of $k$. This result extends to the case where the $\left\{\varepsilon_{t}\right\}$ are a martingale difference series with conditional heteroscedasticity (see, for example, Campbell, Lo and MacKinlay 1997), though the variance $\sigma_{k}^{2}$ has to be adjusted a little. The fixed $k$ asymptotic distribution is well known to fare badly when $k$ is large relative to $n$. (See Lo and MacKinlay, 1989).

As an alternative to the fixed $k$ asymptotics, Richardson and Stock (1989) develop an alternative approach under $k \rightarrow \infty, n \rightarrow \infty$ and $k / n \rightarrow \delta>0$ asymptotics. Under the assumption that $\left\{x_{t}\right\}$ follows (1), where $\left\{\varepsilon_{t}\right\}$ is a conditionally heteroscedastic martingale difference series, they show that

$$
V R(k) \stackrel{D}{\rightarrow}{\frac{1}{(1-\delta)^{2} \delta}}_{\delta}^{\mathrm{Z}_{1}}(W(\lambda)-W(\lambda-\delta)-\delta W(1))^{2} d \lambda
$$

where $W(\lambda)$ is standard Brownian motion. Thus, while the $V R$ statistic converges to 1 under the fixed $k$ treatment, here it has a nondegenerate limiting distribution. Clearly this distribution is nonnormal and instead can be characterised by a functional of Brownian motions. Through Monte Carlo simulations, Richardson and Stock (1989) demonstrated that this alternate theory provides a much better approximation to the small sample distribution of the $V R$ statistic. 
Of course, the problems of generating consistent tests when the statistic converges to a nondegenerate distribution are well known. In fact, as we show in Theorem 1 below, under a wide class of mean reverting models, the $V R$ statistic converges to a positive multiple of the limiting distribution in (2) when $k / n \rightarrow \delta>0$. This implies that the resulting test based on the $V R$ statistic will be inconsistent for such alternatives when $k$ is a constant fraction of the sample size $n$. In practical terms, this means that no matter how large the sample size, the probability of detecting such an alternative is bounded below some number strictly less than one, which is clearly an undesirable property.

Theorem 1 Let $\left\{e_{t}\right\}$ and $\left\{u_{t}\right\}$ be two series of zero mean independent processes with finite variance $\sigma_{e}^{2}$ and $\sigma_{u}^{2}$ respectively which are independent of each other. Define the process $\left\{y_{t}\right\}$ by $y_{t}=\lambda y_{t-1}+u_{t}$ where $|\lambda|<1$ Let $r_{t}=\mu+r_{t-1}+e_{t}$ and

$$
x_{t}=r_{t}+y_{t}
$$

If $k \rightarrow \infty, n \rightarrow \infty$ and $k / n \rightarrow \delta>0$, then

$$
V R(k) \stackrel{D}{\rightarrow} \frac{(1+\lambda) \sigma_{e}^{2}}{(1+\lambda) \sigma_{e}^{2}+2 \sigma_{u}^{2}}{\frac{1}{(1-\delta)^{2} \delta}}_{\delta}^{\mathrm{Z}_{1}}(W(\lambda)-W(\lambda-\delta)-\delta W(1))^{2} d \lambda
$$

where $W(\lambda)$ is standard Brownian motion.

\section{Proof of Theorem 1:}

Let $U_{t}=x_{t}-x_{t-k}-k \hat{\mu}={ }_{j=t-k+1}^{\mathrm{P}} e_{j}-k \bar{e}+y_{t}-y_{t-k}-k n^{-1}\left(y_{n}-y_{0}\right)$. Thus,

$$
\frac{1}{\sqrt{n}} U_{[n \alpha]}=\frac{1}{\sqrt{n}}{ }_{j=1}^{\left[x^{\alpha]}\right.} e_{j}-\frac{1}{\sqrt{n}}{ }_{j=1}^{\left[n \left(x^{-\delta)]}\right.\right.} e_{j}-\frac{k}{n} \frac{1}{\sqrt{n}}_{j=1}^{X^{n}} e_{j}+\frac{1}{\sqrt{n}}^{3} y_{[n \alpha]}-y_{[n(\alpha-\delta)]}-\frac{k}{n} \frac{y_{n}-y_{0}}{\sqrt{n}}
$$

By (A.6) on page 346 of Richardson and Stock(1989), for $\alpha>\delta$,

$$
\frac{1}{\sqrt{n}}{ }_{j=1}^{[x \alpha]} e_{j}-\frac{1}{\sqrt{n}}{ }_{j=1}^{\left[n \left(x^{-\delta)]}\right.\right.} e_{j}-\frac{k}{n} \frac{1}{\sqrt{n}}{ }_{j=1}^{X^{n}} e_{j} \Rightarrow \sigma_{e}[W(\alpha)-W(\alpha-\delta)-\delta W(1)],
$$


where $W(\alpha)$ is standard Brownian motion. Since $\left\{y_{t}\right\}$ is a stationary process, it is trivially true that

$$
\frac{1}{\sqrt{n}}^{3} y_{[n \alpha]}-y_{[n(\alpha-\delta)]}-\frac{k}{n} \frac{y_{n}-y_{0}}{\sqrt{n}} \Rightarrow 0 .
$$

Hence, by (5), (6) and (7),

$$
\frac{1}{\sqrt{n}} U_{[n \alpha]} \Rightarrow \sigma_{e}[W(\alpha)-W(\alpha-\delta)-\delta W(1)]
$$

Furthermore, by the weak law of large numbers,

$$
\frac{1}{n}_{t=1}^{X^{n}}\left(x_{t}-x_{t-1}-\hat{\mu}\right)^{2} \stackrel{P}{\rightarrow} \sigma_{e}^{2}+\operatorname{Var}\left(y_{t}-y_{t-1}\right)=\sigma_{e}^{2}+\frac{2 \sigma_{u}^{2}}{1+\lambda} .
$$

The same argument as that employed on page 329 of Richardson and Stock(1989) then yields the required result.

Theorem 1 shows that the test based on the $V R$ statistic when $k$ is a fraction of the sample size will result in an inconsistent test for mean reverting alternatives of the form (3). Such mean reverting alternatives are of the kind often imposed in finance applications (See, for example, Poterba and Summers (1988) and Fama and French (1988)). The variable $x_{t}$ contains a permanent (i.e. random walk) component $r_{t}$ and a temporary (i.e. stationary) component $y_{t}$. It is also obvious from (4) that when the variance of the innovations of the temporary component, $\sigma_{u}^{2}$, is small relative to $\sigma_{e}^{2}$ the test based on the $V R$ statistic will have power which is barely larger than the nominal size of the test in detecting the alternative even in infinitely large sample sizes.

A limiting result for $V R$ similar to (4) in Theorem 1 can actually be obtained under much more general conditions on $\left\{r_{t}\right\}$ and $\left\{y_{t}\right\}$. One can allow both the first difference series $\left\{r_{t}-r_{t-1}\right\}$ and $\left\{y_{t}\right\}$ to be arbitrary stationary processes instead of the independent process $\left\{e_{t}\right\}$ and the autoregressive process of order 1 respectively that was imposed in Theorem 1 . Thus, the $V R$ statistic can be shown to be inconsistent for a very wide range of alternatives which might be of interest in detecting mean reversion if $k$ is a fraction of the sample size. 


\section{Power Implications}

The theorem in Section 2 above shows that the VR statistic is inconsistent against meaningful alternatives as $k \rightarrow \infty, n \rightarrow \infty$ and $k / n \rightarrow \delta>0$. Moreover, equation (4) provides the form for the asymptotic power function. In this section, we explore the implications of these results for the VR statistic's power.

As a first step, Table 1 reports the small sample power of the VR statistic for the relevant case in which its power is consistent. In particular, we consider the alternative model described in Theorem 1 under the assumption that $\sigma_{e}^{2}=0$, that is, there is no permanent component to $x_{t}$. As seen from the table, as $n$ increases, the power approaches $100 \%$. Interestingly, this increase is greater for smaller $\delta$ which is consistent with Theorem 1's main implication that, for consistency, $k / n \rightarrow 0$. Intuitively, in small samples, even consistent alternatives are hard to distinguish and the large $k$ problem is attenuated.

Table 2 reports the relevant power results for a variety of alternatives described by Theorem 1 . Several observations are in order. First, the table reports small sample power for $n$ ranging from 60 through 2880 observations, as well as the asymptotic power level. As Theorem 1 shows, and Table 2 demonstrates, the small sample power of the VR statistic is clearly limited, supporting the inconsistency of it as a test statistic. Second, even though there is some increase in power as $n$ increases, the gains are very limited for alternative specifications with substantial permanent components. For example, when the share of the variance captured by the mean-reverting component, $\gamma$, is only 0.25 and 0.5 , respectively, the power is $14 \%$ and $38 \%$ as $n \rightarrow \infty$. Third, these power gains are worse for larger $\delta$, which is consistent with the requirement that $k / n \rightarrow 0$. As an illustration, for $\gamma$ equal to 0.25 , 0.5 and 0.75 , respectively, the power is $14 \%, 38 \%$ and $86 \%$ for $\delta=1 / 6$ versus only $11 \%, 27 \%$ and $62 \%$ for $\delta=1 / 3$. To get a more complete picture of this point, Figure 1 graphs the three-dimensional relation between power, $\gamma$ and $\delta$. As borne out by the figure, lower $\delta^{\prime}$ s increase power, with varying 
impact depending on the permanent component's importance (i.e., level of $\gamma$ ). For a fixed value of $\gamma$, the drop-off in the power, as $\delta$ increases, is quite dramatic. Finally, note that the mean-reversion parameter, $\lambda$, has no effect upon the asymptotic distribution as the VR statistic is consistent against pure mean reversion alternatives (e.g., see Table 1). Table 2, however, shows that $\lambda$ is important in small samples.

On the one hand, these results are fairly discouraging for developing tests against slow mean reverting alternatives. Given a fixed $\delta$, the VR statistic will have limited success. Within the Richardson and Stock (1989) framework, however, Perron and Vodounou (2001) do manage to characterize the maximal possible power by taking a continuous-time limit given a fixed data span, $n$. On the other hand, and in contrast to the Perron and Vodounou (2001) result, Theorem 1 suggests an alternative approach in which $k$ is allowed to increase with $n$ but at a slower rate. This guarantees consistency against a wide range of alternatives. Practically, this means that, for power purposes, $k$ cannot be too large in small samples. The practical issue of how to then adjust the size of the statistics for a given $k$ in small samples remains a question for future research.

\section{References}

Campbell, J., Lo, A. and MacKinlay, A. (1997). The Econometrics of Financial Markets. Princeton University Press, Princeton.

Campbell, J. and Mankiw, N. G. (1987). “Are Output Fluctuations Transitory?”, Quarterly Journal of Economics, 102, 857-880.

Cochrane, J. (1988). "How Big is the Random Walk in GNP?", Journal of Political Economy, 96, 893-920.

Daniel, K. (2001). "The Power and Size of Mean Reversion Tests", forthcoming, Journal of Empirical Finance.

Fama, E. and French, K. (1988). "Permanent and Temporary Components of Stock Prices”, Journal 
of Political Economy”, 96, 246-273.

Faust, J. (1992). “When Are Variance Ratio Tests For Serial Dependence Optimal?”, Econometrica, $60,1215-1226$.

Lo, A. and MacKinlay, A. C. (1989). The Size and Power of the Variance Ratio Test in Finite Samples: a Monte Carlo Investigation. Journal of Econometrics, 40, 203-238.

Lo, A. and MacKinlay, A. C. (1988). "Stock Market Prices Do Not Follow Random Walks: Evidence from a Simple Specification Test", Review of Financial Studies, 1,41-66.

Perron, P. and Vodounou, C. (2001). "The Variance Ratio Test: An Analysis of Size and Power based on a Continuous-Time Asymptotic framework", working paper, Boston University.

Poterba, J. and Summers, L. (1988). "Mean Reversion in Stock returns: Evidence and Implications", Journal of Financial Economics, 22, 27-60.

Richardson, M and Smith, T. (1994). "A Unified Approach to Testing for Serial Correlation in Stock Returns", Journal of Business, 67, 371-399.

Richardson, M and Smith, T. (1991). “Tests of Financial Models with the Presence of Overlapping Observations", Review of Financial Studies, 4, 227-254.

Richardson, M. and Stock, J. (1989). "Drawing Inferences from Statistics based on Multi-Year Asset Returns", Journal of Financial Economics, 25, 323-348. 


\section{Figure 1: Asymptotic Power versus Gamma and Delta}

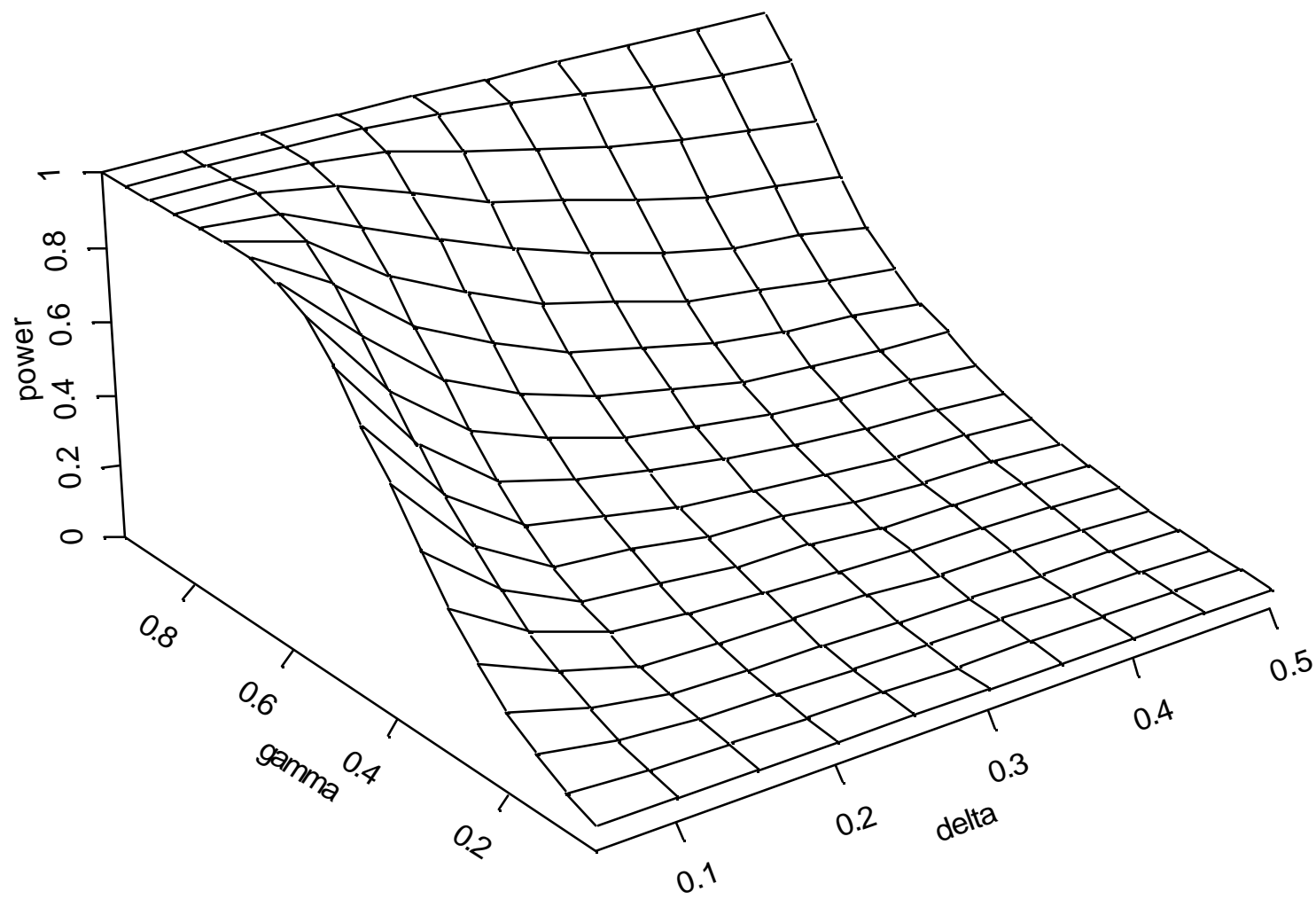

This figure reports the asymptotic power of the variance-ratio statistic for a test of the random walk null against a specific alternative model at the $5 \%$ level of significance for a range of $\delta$ 's and $\gamma$ 's, where $\delta$ represents the ratio of the variance horizon to the data length and $\gamma$ represents the proportion of the variance due to the mean-reverting component. In particular, the alternative model is $x_{t}=r_{t}+y_{t}$ where $r_{t}=r_{t-1}+e_{t}$, $\mathrm{y}_{\mathrm{t}}=\lambda \mathrm{y}_{\mathrm{t}-1}+\mathrm{u}_{\mathrm{t}}$ and $\gamma=\frac{2 \sigma_{u}^{2}}{(1+\lambda) \sigma_{e}^{2}+2 \sigma_{u}^{2}}$. 
Table 1: This table reports the power of the variance-ratio statistic for a one tailed test of the random walk null against a variety of alternatives at the 5\% level of significance for $\delta=1 / 6$ and $\delta=1 / 3$, where $\delta$ represents the ratio of the variance horizon to the data length. The alternative model is $\mathrm{x}_{\mathrm{t}}=\mathrm{y}_{\mathrm{t}}$ where $\mathrm{y}_{\mathrm{t}}=\lambda \mathrm{y}_{\mathrm{t}-1}+\mathrm{u}_{\mathrm{t}}$ and $\sigma_{\mathrm{u}}^{2}=1$.

\begin{tabular}{|c|c|c|c|}
\hline $\mathrm{n} ; \delta=1 / 6$ & $\lambda=0.9$ & $\lambda=0.95$ & $\lambda=0.98$ \\
\hline 60 & 7 & 4 & 3 \\
120 & 21 & 8 & 4 \\
180 & 47 & 11 & 4 \\
360 & 98 & 42 & 8 \\
720 & 100 & 94 & 22 \\
1440 & 100 & 100 & 69 \\
2880 & 100 & 100 & 98 \\
\hline $\mathrm{n} ; \delta=1 / 3$ & $\lambda=0.9$ & $\lambda=0.95$ & $\lambda=0.98$ \\
\hline 60 & 7 & 4 & 3 \\
120 & 21 & 8 & 4 \\
180 & 41 & 11 & 5 \\
360 & 90 & 34 & 19 \\
720 & 100 & 79 & 47 \\
1440 & 100 & 98 & 78 \\
2880 & 100 & 100 & \\
\hline
\end{tabular}


Table 2: This table reports the power of the variance-ratio statistic for a one tailed test of the random walk null against a variety of alternatives at the $5 \%$ level of significance for $\delta=1 / 6$ and $\delta=1 / 3$, where $\delta$ represents the ratio of the variance horizon to the data length. The alternative model is $\mathrm{x}_{\mathrm{t}}=\mathrm{r}_{\mathrm{t}}+\mathrm{y}_{\mathrm{t}}$ where $\mathrm{r}_{\mathrm{t}}=\mathrm{r}_{\mathrm{t}-1}+\mathrm{e}_{\mathrm{t}}, \mathrm{y}_{\mathrm{t}}=\lambda \mathrm{y}_{\mathrm{t}-1}+\mathrm{u}_{\mathrm{t}}$ and

$$
\gamma=\frac{2 \sigma_{u}^{2}}{(1+\lambda) \sigma_{e}^{2}+2 \sigma_{u}^{2}}
$$

\begin{tabular}{|c|ccc|ccc|ccc}
\hline$\delta=1 / 6$ & & $\lambda=0.9$ & \multicolumn{3}{c|}{$\lambda=0.95$} & \multicolumn{3}{c}{$\lambda=0.98$} \\
$\mathrm{n}$ & $\gamma=0.25$ & $\gamma=0.5$ & $\gamma=0.75$ & $\gamma=0.25$ & $\gamma=0.5$ & $\gamma=0.75$ & $\gamma=0.25$ & $\gamma=0.5$ & $\gamma=0.75$ \\
\hline 60 & 4 & 5 & 6 & 4 & 5 & 4 & 4 & 3 & 4 \\
120 & 6 & 9 & 14 & 6 & 4 & 6 & 3 & 4 & 3 \\
180 & 9 & 12 & 23 & 6 & 7 & 9 & 6 & 4 & 3 \\
360 & 9 & 21 & 51 & 7 & 13 & 23 & 6 & 5 & 5 \\
720 & 12 & 28 & 69 & 10 & 21 & 48 & 5 & 8 & 12 \\
1440 & 13 & 32 & 78 & 11 & 25 & 69 & 9 & 14 & 30 \\
2880 & 16 & 37 & 83 & 14 & 30 & 81 & 10 & 26 & 55 \\
$\infty$ & 14 & 38 & 86 & 14 & 38 & 86 & 14 & 38 & 86 \\
\hline$\delta=1 / 3$ & & $\lambda=0.9$ & & & $\lambda=0.95$ & & & $\lambda=0.98$ & \\
$\mathrm{n}$ & $\gamma=0.25$ & $\gamma=0.5$ & $\gamma=0.75$ & $\gamma=0.25$ & $\gamma=0.5$ & $\gamma=0.75$ & $\gamma=0.25$ & $\gamma=0.5$ & $\gamma=0.75$ \\
\hline 60 & 5 & 5 & 6 & 5 & 5 & 5 & 4 & 4 & 4 \\
120 & 6 & 9 & 11 & 6 & 5 & 6 & 4 & 4 & 4 \\
180 & 8 & 11 & 18 & 5 & 6 & 9 & 4 & 4 & 3 \\
360 & 7 & 15 & 33 & 5 & 10 & 18 & 5 & 5 & 4 \\
720 & 10 & 21 & 47 & 8 & 15 & 35 & 6 & 7 & 11 \\
1440 & 12 & 24 & 55 & 10 & 18 & 43 & 7 & 11 & 19 \\
2880 & 13 & 25 & 60 & 9 & 21 & 56 & 9 & 18 & 34 \\
$\infty$ & 11 & 27 & 62 & 11 & 27 & 62 & 11 & 27 & 62 \\
\hline
\end{tabular}

